Rodrigo de Barros Paes

\title{
Regulando a Interação de Agentes em Sistemas Abertos - uma Abordagem de Leis
}

DISSERTAÇÃO DE MESTRADO

DEPARTAMENTO DE INFORMÁTICA

Programa de Pós-graduação em

Informática 


\section{Pontificia Universidade $\mathrm{C}_{\text {atólica }}$

Rodrigo de Barros Paes

Regulando a Interação de Agentes em Sistemas Abertos - uma Abordagem de Leis

Dissertação apresentada como requisito parcial para obtenção do grau de Mestre pelo Programa de Pós-graduação em Informática do Departamento de Informática da PUCRio

Orientador: Prof. Carlos José Pereira de Lucena 


\title{
Pontificia Universidade $\mathrm{C}_{\text {atólica }}$

Rodrigo de Barros Paes

\section{Regulando a Interação de Agentes em Sistemas Abertos - uma Abordagem de Leis}

Dissertação apresentada como requisito parcial para obtenção do grau de Mestre pelo Programa de Pós-graduação em Informática do Departamento de Informática do Centro Técnico Científico da PUC-Rio.Aprovada pela Comissão Examinadora abaixo assinada.

\author{
Prof. Carlos José Pereira de Lucena \\ Orientador \\ Departamento de Informática — PUC-Rio
}

Prof. Bruno Feijó PUC-Rio

Prof. Simone Barbosa

PUC-Rio

Prof. José Eugenio Leal Coordenador Setorial do Centro Técnico Científico -

PUC-Rio

Rio de Janeiro, 18 de Março de 2005 
Todos os direitos reservados. É proibida a reprodução total ou parcial do trabalho em autorização da universidade, do autor e do orientador.

Rodrigo de Barros Paes

Ficha catalográfica

Paes, Rodrigo de Barros

Regulando a interação de agentes em sistemas abertos : uma abordagem de leis / Rodrigo de Barros Paes ; orientador: Carlos José Pereira de Lucena. Rio de Janeiro : PUC-Rio, Departamento de Informática, 2005.

119 f. : il. ; $30 \mathrm{~cm}$

Dissertação (mestrado) - Pontifícia Universidade Católica do Rio de Janeiro, Departamento de Informática.

Inclui referências bibliográficas

1. Informática - Teses. 2. Sistemas multi-agentes. 3. Interação. 4. Sistemas normativos. 5. Sistemas abertos. 6. Leis. I. Lucena, Carlos José Pereira de. II. Pontifícia Universidade Católica do rio de Janeiro. Departamento de Informática. III. Título.

CDD: 004 


\section{Agradecimentos}

Ao professor Lucena pelos inúmeros ensinamentos científicos, pelas oportunidades que me foram concedidas, pelo exemplo de caráter e de competência profissional.

Ao professor Paulo Alencar pelo apoio que recebi no Canadá, além das várias e proveitosas discussões sobre este trabalho e sobre trabalhos futuros.

Ao professor Don Cowan também pelo grande apoio que me ofereceu no Canadá.

Ao Guga pela amizade, pelos conselhos, pela paciência nas nossas discussões e pela contribuição neste trabalho.

Ao Hyggo pelas contribuições a este trabalho, pela amizade mas, principalmente, pelas nossas conversas, muitas vezes filosofais.

Aos meus amigos do LES, Gaúcho, Alessandro Véio, Uirá, Baiano, Viviane, Choren, por terem tornado divertidos estes dois anos no Rio e, é claro, por terem se tornado meus amigos.

A todos os outros amigos do LES pela convivência que me proporcionou muito aprendizado.

Ao Choren por ter revisado e ajudado com sugestões para os artigos que escrevemos.

Ao apoio e corujice incondicional da minha vovó Léa.

Ao também incondicional apoio do meu Pai.

A todas as minhas tias.

A todos os professores que contribuíram para a minha formação.

A CAPES pelo apoio financeiro. 


\section{Resumo}

Paes, Rodrigo; Lucena, Carlos José Pereira de. Regulando a Interação de Agentes em Sistemas Abertos - uma Abordagem de Leis. Rio de Janeiro, 2005. 119p. Dissertação de Mestrado — Departamento de Informática, Pontifícia Universidade Católica do Rio de Janeiro.

Nesta dissertação, apresenta-se uma abordagem para regular a interação dos agentes que fazem parte de um sistema multi-agente aberto. Em sistemas abertos, os agentes podem ser não-cooperativos, estão imersos em um ambiente altamente imprevisível e, freqüentemente, os outros agentes que compõem o sistema não são conhecidos $a$ priori. Para algumas classes de aplicações, esta imprevisibilidade não é adequada, podendo levar a falhas de software. Desta forma, é proposta uma abordagem baseada em leis de interação para construir sistemas multi-agentes abertos, onde um controle sobre o comportamento dos agentes é esperado. Propõe-se um modelo conceitual para a especificação da forma como as interações são reguladas em um sistema multi-agente. Este modelo conceitual trata conceitos como cenas, normas e restrições de forma integrada. Além disso, a interação entre os agentes deve ser monitorada e as leis que foram especificadas devem ser aplicadas. Para isto, propõe-se uma linguagem declarativa para a especificação da interação de acordo com os elementos do modelo conceitual e uma infra-estrutura de software que age como mediador das interações garantindo que elas estejam de acordo com as especificações.

\section{Palavras-chave}

Sistemas multi-agentes. Interação. Sistemas normativos. Sistemas abertos. Leis. 


\section{Abstract}

Paes, Rodrigo; Lucena, Carlos José Pereira de. Regulating Agent's Interaction - a Law Enforcement Approach. Rio de Janeiro, 2005. 119p. MSc. Dissertation — Departamento de Informática, Pontifícia Universidade Católica do Rio de Janeiro.

In this work, we propose an approach for regulating agents' interaction on an open multi-agent system. In open systems, agents are immersed in a highly unpredictable environment, they can be self-interested, and other agents are frequently unknown beforehand. We argue that, in some applications, unexpected behavior may lead to system faults. For this reason, we propose a law enforcement approach to build open multi-agent systems where a certain degree of control over agents' behavior is desirable. A conceptual model is proposed to specify how the interactions of an open multi-agent system should happen. This model deals with concepts such as norms, constraints and scenes in a integrated way. We also propose a declarative language that allows the interaction's specification according to the elements that compose the conceptual model, and a software infrastructure that acts as a mediator monitoring and enforcing agents' interaction.

\section{Keywords}

Multi-agent systems; Interaction; Normative systems; Open Systems; Laws. 


\section{Conteúdo}

1 Introdução 11

\begin{tabular}{lll}
\hline 1.1 & Definição do Problema & 11
\end{tabular}

$\begin{array}{lll}1.2 & \text { Solução proposta } & 12\end{array}$

$\begin{array}{lll}1.3 & \text { Contribuições } & 13\end{array}$

\begin{tabular}{lll}
\hline 1.4 & Organização da Dissertação & 14
\end{tabular}

2 Fundamentos $\quad 15$

2.1 Sistemas Abertos 15

$\begin{array}{lll}2.2 & \text { Sistemas Multi-Agentes } & 16\end{array}$

3 Trabalhos Relacionados $\quad 21$

3.1 Abordagem Interagent para o desenvolvimento de agentes 21

3.2 Instituições Eletrônicas 22

3.3 Normas abstratas, estatutos e instituições eletrônicas 25

3.4 Agentes deliberativos: princípios e arquitetura 26

3.5 Adoção de normas em um agente deliberativo 27

3.6 A abordagem LGI - Law-Governed Interaction 28

$\begin{array}{lll}3.7 & \text { Outros trabalhos } & 29\end{array}$

4 Modelo Conceitual $\quad \mathbf{3 4}$

4.1 Eventos: relacionando os conceitos 34

4.2 Agente e Mensagem 35

4.3 Protocolo, Estado e Transição 37

\begin{tabular}{lll}
\hline 4.4 & Relógio & 39
\end{tabular}

4.5 Norma 40

4.6 Cena 41

$\begin{array}{lll}4.7 & \text { Navegando entre as Cenas } & 43\end{array}$

4.8 LawOrganization 45

4.9 Restrições 46

4.10 Ações 47

5 Suporte de Software 449

5.1 Modelo de Interação 50

5.2 Módulo de Comunicação $\quad 52$

5.3 Agente Mediador $\quad 56$

5.4 Módulo de Suporte ao Desenvolvedor de Agentes 70

6 Estudo de caso: Aeroporto $\quad \mathbf{8 3}$

6.1 Utilizando os conceitos do modelo conceitual 84

7 Discussões e trabalhos futuros $\quad 95$

$\begin{array}{lr}\text { Referências Bibliográficas } & 98\end{array}$ 
$\begin{array}{ll}\text { A A Lei do Aeroporto } & 106\end{array}$

B Gramática do XMLaw 111

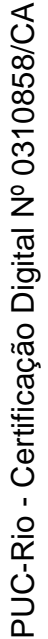




\section{Lista de Figuras}

4.1 Modelo Conceitual $\quad 35$

4.2 Cadeia de Eventos 35

4.3 Modelo Conceitual: Agente e Mensagem 36

4.4 Modelo Conceitual: Foco no Protocolo 37

4.5 Ciclo de Vida do Relógio $\quad 40$

$\begin{array}{lll}4.6 & \text { Atividades de uma Ação } & 48\end{array}$

5.1 Framework: Módulos e Desenvolvedores 51

5.2 Modelo de Interação para a Aplicação das Leis 51

5.3 Camada de Comunicação 54

5.4 Módulo de Mensagens 56

5.5 Atividades do mediador 57

5.6 Tipos de Eventos $\quad 58$

5.7 IEvent Interface 58

5.8 Problema com a Implementação Usual da Notificação de Eventos 60

5.9 Observer e Subject 61

5.10 Módulo de Triggers 63

5.11 Classe Context 64

5.12 Módulo de Relógio 65

5.13 Normas 66

5.14 Módulo de Protocolo 67

5.15 Atividades de uma Transição 68

5.16 Módulo de Cenas 69

5.17 Módulo de Restrições $\quad 69$

5.18 Módulo de Ações 71

5.19 Protocolo do Mediador - Mensagens que os Agentes Podem Enviar 73

5.20 Protocolo do Mediador - Mensagens que os Mediadores Podem $\begin{array}{ll}\text { Enviar } & 73\end{array}$

$\begin{array}{ll}5.21 \text { Classe Agent } & 74\end{array}$

6.1 Cenas Modularizando a Interação 84

6.2 Símbolos Utilizados para Representar as Cenas 85

6.3 Leis da Cena chegada 85

6.4 Cena: chegada $\quad 86$

$\begin{array}{lll}6.5 & \text { Leis da cena seleção } & 87\end{array}$

6.6 Cena: seleção $\quad 87$

6.7 Leis da cena negociação $\quad 89$

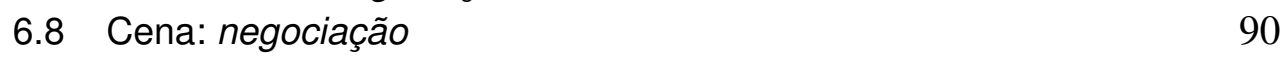

6.9 Leis da cena pagamento 93

6.10 Cena: pagamento 93 
Most brilliant ideas happen when you are not thinking about your problem at all.

Anonymous, . 